University of New Hampshire

University of New Hampshire Scholars' Repository

Physics Scholarship

Physics

$6-2003$

\title{
Suprathermal electron isotropy in high-beta solar wind and its role in heat flux dropouts
}

\author{
N. U. Crooker \\ D. E. Larson \\ S. W. Kahler \\ S. M. Lamassa \\ Harlan E. Spence \\ Boston University, harlan.spence@unh.edu
}

Follow this and additional works at: https://scholars.unh.edu/physics_facpub

Part of the Physics Commons

\section{Recommended Citation}

Crooker, N. U., D. E. Larson, S. W. Kahler, S. M. Lamassa, and H. E. Spence (2003), Suprathermal electron isotropy in high-beta solar wind and its role in heat flux dropouts, Geophys. Res. Lett., 30, 1619, doi:10.1029/2003GL017036, 12.

This Article is brought to you for free and open access by the Physics at University of New Hampshire Scholars' Repository. It has been accepted for inclusion in Physics Scholarship by an authorized administrator of University of New Hampshire Scholars' Repository. For more information, please contact Scholarly.Communication@unh.edu. 


\title{
Suprathermal electron isotropy in high-beta solar wind and its role in heat flux dropouts
}

\author{
N. U. Crooker, ${ }^{1}$ D. E. Larson, ${ }^{2}$ S. W. Kahler, ${ }^{3}$ S. M. Lamassa, ${ }^{1}$ and H. E. Spence ${ }^{1}$ \\ Received 1 February 2003; revised 20 March 2003; accepted 22 April 2003; published 20 June 2003.
}

[1] Time variations in plasma beta and a parameter which measures isotropy in suprathermal electron pitch angle distributions show a remarkably close correspondence throughout the solar wind. The finding implies that highbeta plasma, with its multiple magnetic holes and sharp field and plasma gradients, is conducive to electron pitch-angle scattering, which reduces heat flux from the Sun without field-line disconnection. Thus the finding impacts our understanding of signatures we use to determine magnetic topology in the heliosphere. INDEX TERMS: 2164 Interplanetary Physics: Solar wind plasma; 2159 Interplanetary Physics: Plasma waves and turbulence; 2134 Interplanetary Physics: Interplanetary magnetic fields. Citation: Crooker, N. U., D. E. Larson, S. W. Kahler, S. M. Lamassa, and H. E. Spence, Suprathermal electron isotropy in high-beta solar wind and its role in heat flux dropouts, Geophys. Res. Lett., 30(12), 1619, doi:10.1029/2003GL017036, 2003.

\section{Introduction}

[2] Suprathermal electrons $(\mathrm{E}>\sim 80 \mathrm{eV})$ in the solar wind carry heat away from the Sun. As they expand into the heliosphere, the decrease in magnetic field strength decreases their pitch angles, resulting in a focused beam of electrons, or strahl, directed away from the Sun along the magnetic field. The width of the strahl is variable, however [e.g., Hammond et al., 1996], and sometimes the pitch angle distribution is nearly isotropic, with no focused beam and greatly reduced heat flux. These events, called "heat flux dropouts (HFDs)," were interpreted by McComas et al. [1989] as signatures of magnetic field lines no longer connected to the Sun. Later Fitzenreiter and Ogilvie [1992] and Lin and Kahler [1992] challenged that interpretation. They demonstrated that many of the HFDs identified by McComas et al. [1989] were confined to energies below $500 \mathrm{eV}$ and that, in cases with sufficient $>2 \mathrm{keV}$ electron flux, these higher-energy particles continued to stream away from the Sun along what must have been connected field lines. While these challenges undermine the idea that disconnected field lines are common in the solar wind, the issues of what causes HFDs and what fraction of HFDs actually do signal disconnected fields remain unresolved. HFD issues are important because their resolution will have far-reaching implications for the heliospheric magnetic flux budget [e.g., McComas, 1995; Crooker et al.,

\footnotetext{
${ }^{1}$ Center for Space Physics, Boston Univ., Boston, Massachusetts, USA USA.

${ }^{2}$ Space Sciences Laboratory, Univ. of California, Berkeley, California,

${ }^{3}$ Air Force Research Laboratory, Hanscom Air Force Base, Massachusetts, USA.
}

Copyright 2003 by the American Geophysical Union. 0094-8276/03/2003GL017036
2002] and will help discriminate between proposed mechanisms for the 11-year reversal of the solar magnetic field [e.g., Wang et al., 2000; Fisk and Schwadron, 2001]. This paper addresses the cause of HFDs. The findings reported here must be taken into account in any model of HFDs.

[3] Historically there has been a close relationship between HFDs and the heliospheric current sheet (HCS). Pilipp et al. [1987] noted that suprathermal electron populations tend to become isotropic there, and McComas et al. [1989] noted that HFDs often coincide with or occur near the HCS. In a case study of an HCS embedded in an HFD, Crooker et al. [1996] found that the HFD coincided with a series of plasma sheets and proposed that the structure peculiar to plasma sheets, rather than the current sheet itself, may be responsible for HFDs. Plasma sheets, as defined by Winterhalter et al. [1994], are high-beta, high-density structures surrounding the HCS, where beta is the ratio of gas to magnetic pressure. This paper confirms that HFDs are related to plasma sheets, most of which surround current sheets, whether the current sheets are the HCS or localized structures [Crooker, 2003]. Moreover, this paper generalizes the HFDplasma sheet relationship to include all high-beta structures.

[4] There are two aspects to consider when addressing the cause of an HFD. Heat flux drops both when the total suprathermal electron flux integrated over pitch angle drops and when the suprathermal electron distribution becomes isotropic. In the case of field-line disconnection from the Sun, one expects a decrease in total flux owing to a cutoff of the source of the strahl, leaving a remnant isotropic halo population of presumably back-scattered strahl electrons [e.g., Gosling et al., 2001]. Pitch angle scattering alone, however, may ultimately be responsible for both isotropization and a decrease in total flux. While pitch angle scattering itself does not decrease flux, it may lead to conditions which create a barrier to free outflow of strahl electrons [R. P. Lin, private communication, 2002]. The results reported here, although not decisive on this issue, favor the scattering option, since they show a close relationship between the degree of isotropy and an in situ characteristic of the plasma (i.e., plasma beta).

\section{Case Studies}

[5] The close relationship between isotropy and ion beta is illustrated in Figure 1a with Wind data from March 17-18, 1995. The top panel shows an 18-hour interval of the colorcoded pitch angle spectra (every $1.6 \mathrm{~min}$ at $320 \mathrm{eV}$ ). The yellow and red traces first at the top $\left(180^{\circ}\right)$ and then at the bottom $\left(0^{\circ}\right)$ of the spectrogram indicate strahl electrons directed antiparallel and parallel to the magnetic field, respectively. Since the strahl is always directed away from the Sun along magnetic field lines, independent of how contorted those field lines might be, the spectrogram shows passage from a toward sector to an away sector. The exact 

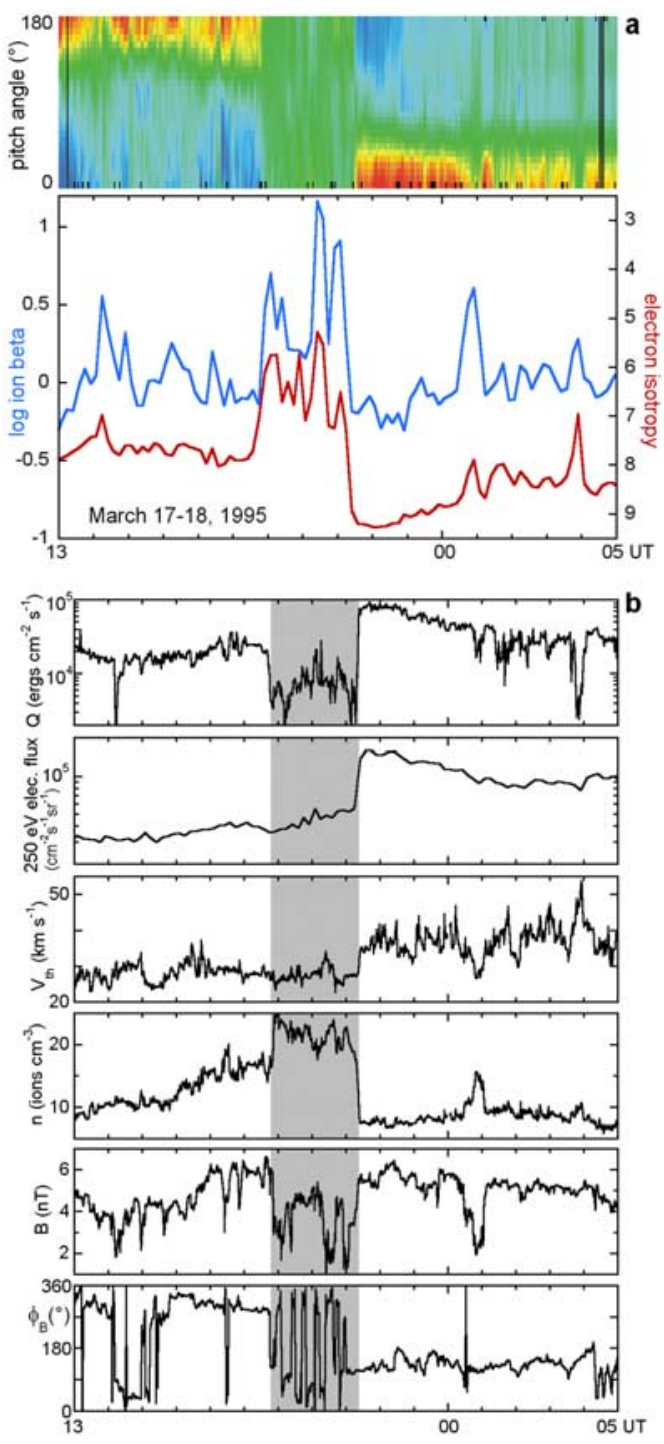

Figure 1. Time variations of Wind plasma and field parameters across a plasma sheet at a sector boundary.

time of sector boundary passage is unknown, however, because the strahl disappears between the sectors and the distribution becomes nearly isotropic. The isotropic interval, roughly 2.5 hours long, matches an interval of elevated beta in the second panel, plotted in blue. The interval thus marks the passage of a heliospheric plasma sheet surrounding the HCS.

[6] The correspondence between plasma sheets and pitch angle isotropy illustrated in Figure 1a led us to search for a more general relationship between beta and isotropy. To easily survey the data, we devised a simple parameter related to variance that represents the degree of anisotropy: $\log \Sigma\left(j_{i}-\langle j\rangle\right)^{2}$, where the sum $\Sigma$ is over 13 pitch angle bins $i$ of 10-min averages of electron flux $j$ (at $250 \mathrm{eV}$ ). The sum is zero for a purely isotropic distribution, which has no variation of flux with pitch angle, and it is large when the strahl is pronounced, with fluxes much higher near $0^{\circ}$ or $180^{\circ}$ than elsewhere. The anisotropy parameter is plotted inversely in red below ion beta in Figure 1a. It thus becomes an isotropy parameter that matches the sense of the variations in beta. The correspondence between the two param- eters is close not only across the plasma sheet but in some of the smaller-scale variations, as well. The correlation coefficient from a scatter plot of the 10-min averages over the 18 -hour interval is 0.7 . The correlation cannot be an instrumental effect because the parameters were calculated with data from different instruments, beta from ions (thus including only partial gas pressure) measured by the Solar Wind Experiment (SWE) [Ogilvie et al., 1995] and isotropy from electrons measured by the Three-Dimensional Plasma and Energetic Particle Experiment (3DP) [Lin et al., 1995]. The only data common to both are from the Magnetic Field Investigation (MFI) [Lepping et al., 1995].

[7] The interval of isotropy in Figure 1a coincides with an HFD, as expected. The top panel of Figure $1 b$ shows the drop in the heat flux magnitude $Q$, calculated in the frame of reference of the bulk (electron) flow. The HFD's location at the sector boundary is typical of those found by McComas et al. [1989], but the second panel shows that the HFD does not display the commonly observed, coincident drop in flux integrated over pitch angle [J. T. Gosling, private communication, 2002], although the flux does rise after the HFD. Thus isotropization rather than a reduction in flux owing to strahl cut-off appears to be the primary cause of this HFD.

[8] The third, fourth, and fifth panels in Figure 1b show that high density $n$ and low magnetic field magnitude $B$ were responsible for the increase in beta, without any significant change in thermal speed $V_{t h}$, as is typical in plasma sheets. The decrease in magnetic pressure and increase in gas pressure leave plasma sheets roughly in pressure balance with their surroundings. Typical of pressure balance structures, they occur over a range of scale sizes, as evidenced by the dips in $B$ or magnetic holes within the plasma sheet [e.g., Burlaga et al., 1990]. The bottom panel in Figure 1b shows large excursions in the azimuthal angle $\phi_{B}$ of the magnetic field within the plasma sheet. These are exaggerated by the scale break between $0^{\circ}$ and $360^{\circ}$, but the field does pass from the toward to the away Parker-spiral hemisphere several times.

[9] In summary, Figure 1 shows a close correspondence between ion beta and suprathermal electron isotropy, especially across a heliospheric plasma sheet in which both rise substantially. The increase in isotropy there substantially reduces the heat flux so that the plasma sheet is also an HFD. The increase in ion beta in the plasma sheet is caused by an increase in $n$ and a decrease in $B$, with smaller-scale fluctuations characteristic of magnetic holes. The magnetic field within the plasma sheet reverses multiple times, indicating possible polarity inversions.

[10] The coincidence of suprathermal electron isotropy and HFDs with high-beta plasma sheets is not confined to those plasma sheets that obviously carry the global heliospheric current sheet, as in Figure 1, but occurs in all plasma sheets. Plasma sheets are highly variable structures that are often found away from the true polarity reversals identified in electron spectrograms [e.g., Crooker, 2003].

[11] Even more generally, the correspondence between beta and electron isotropy that is so apparent at plasma sheets extends to all high-beta structures, even the pressure balance structures in the high-speed wind, far from sector boundaries [e.g., Reisenfeld et al., 1999]. An example is shown in Figure 2, where the average speed was $636 \mathrm{~km} \mathrm{~s}^{-1}$ compared to $399 \mathrm{~km} \mathrm{~s}^{-1}$ for the interval in Figure 1. Again 

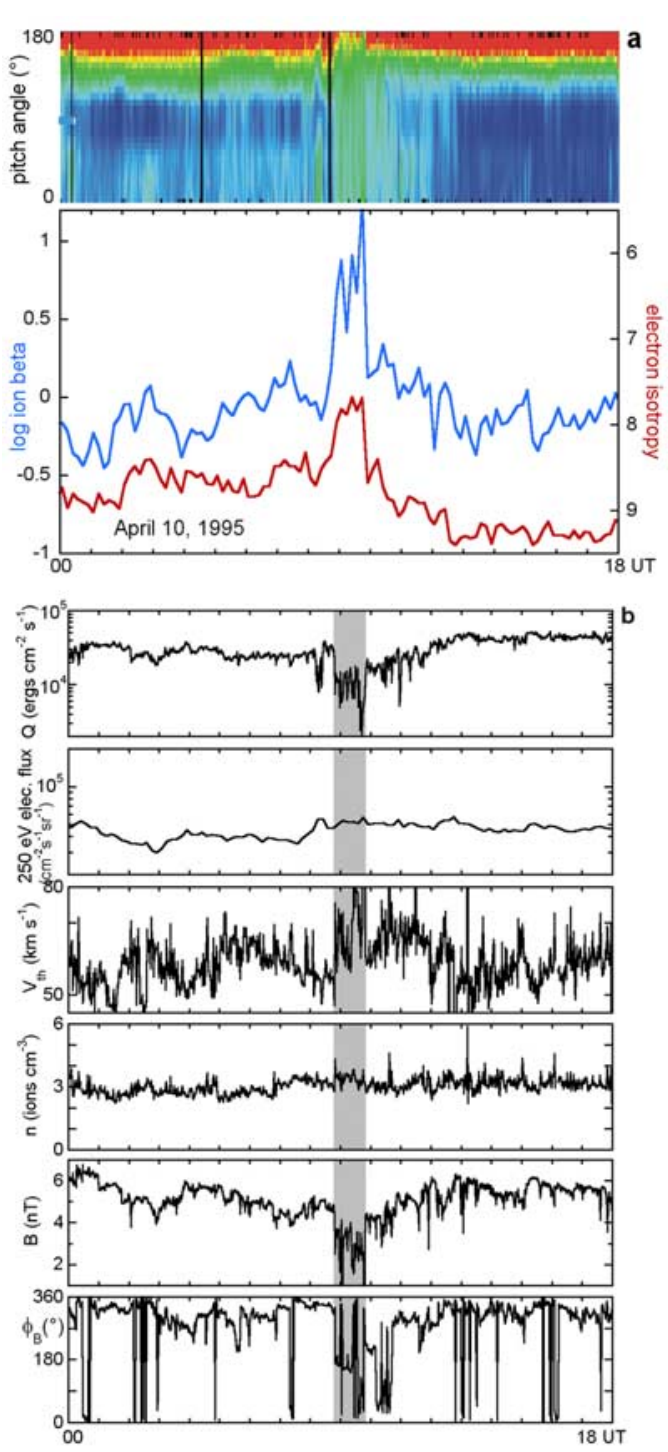

Figure 2. Time variations of Wind plasma and field parameters across a high-beta structure in the high-speed wind.

there is a close correspondence between ion beta and isotropy (Figure 2a), with a correlation coefficient of 0.6 , slightly lower than in Figure 1 because the range of isotropy is smaller. The heat flux dropout (top panel of Figure $2 b$ ), less pronounced than for plasma sheets, reflects only the increase in isotropy, since the integrated flux remained steady (second panel). The hour-long dip in $B$ (fifth panel), replete with small-scale magnetic holes, is balanced in this case by a rise in $V_{t h}$ (third panel), while $n$ (fourth panel) remains relatively constant. The profile of $\phi_{B}$ (bottom panel) shows field reversals within the high-beta structure while the spectrogram (Figure 2a) shows no true polarity reversals, implying fields turned back on themselves. Thus the HFD in the fast wind has the same characteristics as the HFD in the plasma sheet except that elevated $V_{t h}$ rather than $n$ balances the depressed $B$.

\section{Statistics}

[12] The similarity of patterns in Figure 1 and Figure 2 suggests that there should be no systematic difference in the

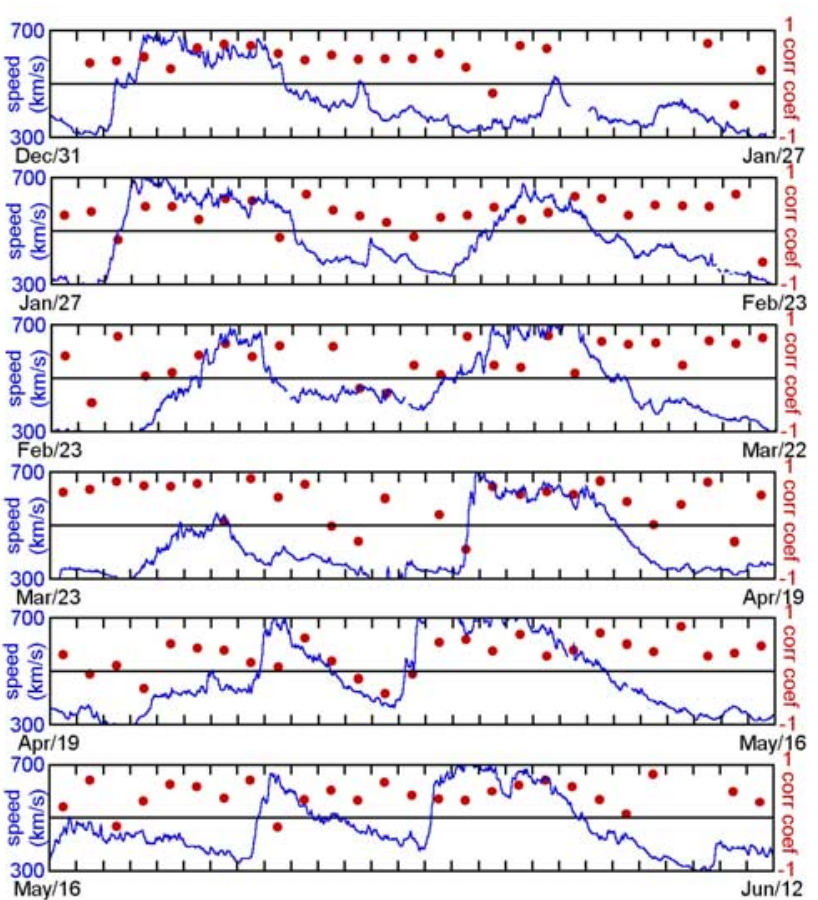

Figure 3. Daily correlation coefficients (dots) between suprathermal electron isotropy and ion beta superposed on 27-day recurrence plots of solar wind speed.

correspondence between beta and isotropy in the fast wind compared to the slow wind. This expectation is confirmed in Figure 3 with data from the first half of 1995, when highspeed streams recurred in the ecliptic plane with each 27-day rotation of the Sun. Superposed on the 27-day recurrence plots of solar wind speed are red dots marking daily correlation coefficients between 10-min averages of log ion beta and the isotropy parameter. The median of the coefficients is 0.44 , and the range is -0.57 to 0.87 . Data from days with poor or negative correlations tend to be structureless in beta on a scale of hours but contain large-scale trends which sometimes anticorrelate. While the coefficients are variable, they show no overall ordering by stream structure.

[13] Figure 4 illustrates the correspondence between isotropy and beta in the form of a contoured log-log scatter plot of modified parameters. Here beta includes both ion

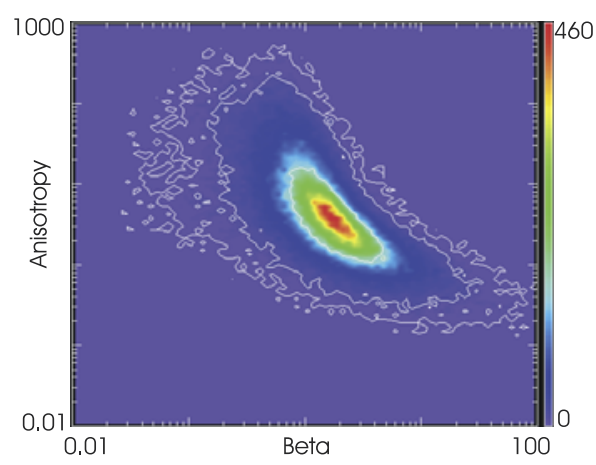

Figure 4. Contoured scatter plot between suprathermal electron anisotropy and plasma beta from Wind 3DP data covering $1 / 1 / 1995$ to $5 / 31 / 2002$. 
and electron pressure, and the isotropy parameter for each pitch angle spectrum is the ratio of maximum to minimum flux minus one, plotted as anisotropy (not inverted). This parameter has the advantage of being dimensionless, like beta. The plot consists of 1.6-min values of $272 \mathrm{eV}$ flux for nearly the entire Wind data set, from 1/1/1995 through 5/31/ 2002. The result shows a clear elongation in the contours of point density, indicating the strong anticorrelation between anisotropy and beta.

\section{Discussion}

[14] The finding reported here, that suprathermal electron isotropy tracks beta in the solar wind, suggests that highbeta plasma is conducive to pitch-angle scattering, possibly by instability-driven wave-particle interactions [e.g., Gary et al., 1999; S. P. Gary, private communication, 2003]. The magnetic holes and multiple field reversals characteristic of the high-beta events illustrated here may be generated by those instabilities. We note that these features have scale sizes comparable to the scale size of the neutral sheet in Earth's magnetotail and thus may de-magnetize electrons in a manner similar to that described by Speiser [1965]. On the other hand, Reames et al. [2001] found a correlation between beta and isotropy in distributions of $\mathrm{MeV}$ ions, implying that the scattering mechanism must be relatively universal.

[15] Whatever the mechanism, it appears that pitch-angle scattering is an important contributor to HFDs and, for the cases shown here, with no drop in integrated flux, the only contributor. Since the degree of pitch-angle isotropy is proportional to beta, a result seen not only statistically but in the gradual flattening of spectra going in to some plasma sheets (not shown), it nearly always must reflect some gradual process like pitch-angle scattering rather than an abrupt process like disconnection. On the other hand, for the numerous HFD cases where integrated flux does drop [J. T. Gosling, private communication, 2002], if disconnection is disallowed as an interpretation, some process in addition to scattering must be postulated to account for the flux drop.

\section{Conclusions}

[16] While suprathermal electron flows have been serving as invaluable tools for sensing open (unidirectional), closed (counterstreaming), and inverted (sunward) field lines in the heliosphere, their usefulness for sensing disconnected fields (dropouts) seems to be seriously compromised by a process which isotropizes their pitch angle distributions. By devising a parameter which distinguishes between isotropy and a drop in integrated flux, both of which contribute to HFDs, and both of which must contribute if the cause is disconnection, we have shown that isotropy alone is correlated with plasma beta throughout the solar wind and sometimes is solely responsible for an HFD. This implies the existence of a process independent of disconnection that scatters electron pitch angles.

[17] Acknowledgments. This research was supported by NASA under grant NAG5-10856 and by NSF under grant ATM0119700.

\section{References}

Burlaga, L. F., J. D. Scudder, L. W. Klein, and P. A. Isenberg, Pressurebalanced structures between $1 \mathrm{AU}$ and $24 \mathrm{AU}$ and their implications for solar wind electrons and interstellar pickup ions, J. Geophys. Res., 95, 2229-2239, 1990.

Crooker, N. U., Heliospheric current and plasma sheet structure, in Solar Wind Ten, edited by M. Velli et al., AIP Conf. Proc., in press, 2003.

Crooker, N. U., M. E. Burton, E. J. Smith, J. L. Phillips, and A. Balogh, Heliospheric plasma sheets as small-scale transients, J. Geophys. Res., 101, 2467-2474, 1996.

Crooker, N. U., J. T. Gosling, and S. W. Kahler, Reducing heliospheric magnetic flux from CMEs without disconnection, J. Geophys. Res., 107(2), 1028, doi:10.1029/2001JA000236, 2002.

Fisk, L. A., and N. A. Schwadron, The behavior of the open magnetic field of the Sun: I, Astrophys. J., 560, 425-438, 2001.

Fitzenreiter, R. J., and K. W. Ogilvie, Heat flux dropouts in the solar wind and Coulomb scattering effects, J. Geophys. Res., 97, 19,213-19,219, 1992.

Gary, S. P., E. Neagu, and R. M. Skoug, Solar wind electrons: Parametric constraints, J. Geophys. Res., 104, 19,843-19,849, 1999.

Gosling, J. T., R. M. Skoug, and W. C. Feldman, Solar wind electron halo depletions at $90^{\circ}$ pitch angle, Geophys. Res. Lett., 28, 4155-4158, 2001.

Hammond, C. M., W. C. Feldman, D. J. McComas, J. L. Phillips, and R. J. Forsyth, Variation of electron-strahl width in the high-speed solar wind: Ulysses observations, Astron. Astrophys., 316, 350-354, 1996.

Lepping, R. L., et al., The Wind magnetic field investigation, Space Sci. Rev., 71, 207-229, 1995.

Lin, R. P., and S. W. Kahler, Interplanetary magnetic field connection to the Sun during electron heat flux dropouts in the solar wind, J. Geophys. Res., 97, 8203-8209, 1992

Lin, R. P., et al., A three-dimensional plasma and energetic particle investigation for the Wind spacecraft, Space Sci. Rev., 71, 125-153, 1995.

McComas, D. J., Tongues, bottles, and disconnected loops: The opening and closing of the interplanetary magnetic field, Rev. Geophys., Suppl., 33, 603-608, 1995.

McComas, D. J., J. T. Gosling, J. L. Phillips, S. J. Bame, J. G. Luhmann, and E. J. Smith, Electron heat flux dropouts in the solar wind: Evidence for interplanetary magnetic field reconnection?, J. Geophys. Res., 94, 6907-6916, 1989.

Ogilvie, K. W., et al., SWE, A comprehensive plasma instrument for the Wind spacecraft, Space Sci. Rev., 71, 55-77, 1995.

Pilipp, W. G., H. Miggenrieder, K.-H. Mülhäuser, H. Rosenbauer, R. Schwenn, and F. M. Neubauer, Variations of electron distribution functions in the solar wind, J. Geophys. Res., 92, 1103-1118, 1987.

Reames, D. V., C. K. Ng, and D. Berdichevsky, Angular distributions of solar energetic particles, Astrophys. J., 550, 1064-1074, 2001.

Reisenfeld, D. B., D. J. McComas, and J. T. Steinberg, Evidence of a solar origin for pressure balance structures in the high-latitude solar wind, Geophys. Res. Lett., 26, 1805-1808, 1999.

Speiser, T. W., Particle trajectories in model current sheets, J. Geophys. Res., 70, 4219-4226, 1965.

Wang, Y. M., N. R. Sheeley Jr., and J. Lean, Understanding the evolution of the Sun's open magnetic flux, Geophys. Res. Lett., 27, 621-624, 2000.

Winterhalter, D., E. J. Smith, M. E. Burton, N. Murphy, and D. J. McComas, The heliospheric plasma sheet, J. Geophys. Res., 99, 6667-6680, 1994.

N. U. Crooker, S. M. Lamassa, and H. E. Spence, Center for Space Physics, Boston Univ., Boston, MA, USA. (crooker@bu.edu)

D. E. Larson, Space Sciences Laboratory, Univ. of California, Berkeley, CA, USA.

S. W. Kahler, Air Force Research Laboratory, Hanscom Air Force Base, MA, USA. 